\title{
STRAIN ULTRASOUND ELASTOGRAPHY IN THE DIAGNOSTIC EVALUATION OF THYROID NODULES
}

\author{
Siderova $\mathbf{M}^{1}$, Hristozov $\mathrm{K}^{1}$, Krasnaliev $\mathbf{I}^{\mathbf{2}}$ \\ ${ }^{1}$ Clinic of Endocrinology, ${ }^{2 D e p a r t m e n t ~ o f ~ P a t h o l o g y, ~ U n i v e r s i t y ~ H o s p i t a l ~ " S t . ~ M a r i n a ", ~ V a r n a, ~ B u l g a r i a ~}$
}

\section{Objectives}

Strain elastography (SE) is a relatively new technique that uses ultrasoundbased imaging to quantify the extent to which tissues deform with the application of pressure.

The aim of the study was to determine different types of thyroid nodules according to their elasticity and to evaluate the diagnostic accuracy of strain elastography in detection of thyroid cancer.

\section{Materials and Methods}

114 thyroid nodules in 84 patients were examined prospectively with conventional Bmode US, color Doppler, strain elastography (SE) and fine needle aspiration biopsy (FNAB). 72 nodules in 50 patients were submitted to surgery and histologically assessed. For final diagnosis we accepted histology in operated cases and cytology for the rest. Study design is presented in Figure 1.

\begin{tabular}{|c|c|c|c|c|c|c|}
\hline \multirow[b]{2}{*}{$\begin{array}{l}\text { Thyroid } \\
\text { nodules } \\
=114\end{array}$} & \multicolumn{6}{|c|}{ Elasticity score } \\
\hline & \multicolumn{2}{|c|}{ Score 1} & Score 2 & Score 3 & Score 4 & Scor \\
\hline $\begin{array}{l}\text { Benign } \\
=76\end{array}$ & \multicolumn{2}{|c|}{$32,9 \%$} & $34,2 \%$ & $26,3 \%$ & $6,6 \%$ & $0 \%$ \\
\hline $\begin{array}{c}\text { Malignant } \\
=38\end{array}$ & \multicolumn{2}{|c|}{$0 \%$} & $5,3 \%$ & $18,4 \%$ & $55,3 \%$ & $21,1 \%$ \\
\hline $\begin{array}{l}\text { p-value } \\
\text { (Fisher's } \\
\text { test) }\end{array}$ & \multicolumn{2}{|c|}{0,0001} & 0,0005 & 0,4839 & 0,0001 & 0,0001 \\
\hline \multicolumn{7}{|c|}{ Table 1. Elastographic characteristics of thyroid nodules } \\
\hline \multicolumn{3}{|c|}{$\begin{array}{l}\text { SN } \\
\%\end{array}$} & $\begin{array}{l}\text { SP } \\
\%\end{array}$ & $\begin{array}{l}\text { PPV } \\
\%\end{array}$ & $\underset{\%}{\operatorname{NPP}} \mathrm{A}$ & $\begin{array}{l}\text { Accuracy } \\
\%\end{array}$ \\
\hline \multicolumn{2}{|c|}{$\begin{array}{l}\text { B-mode US } \\
\text { ( } \geq 3 \text { features of } \\
\text { malignancy) }\end{array}$} & 68,4 & 93,4 & 83,9 & 85,5 & 85,1 \\
\hline \multicolumn{2}{|l|}{ SE } & 76,3 & 93,4 & 85,3 & 88,8 & 87,7 \\
\hline \multicolumn{2}{|l|}{ FNAB } & 90,3 & 81,1 & 62,2 & 96,1 & 83,5 \\
\hline \multicolumn{2}{|c|}{ B-mode + SE } & 89,5 & 86,2 & 79,1 & 94,4 & 89,0 \\
\hline \multicolumn{2}{|c|}{$\begin{array}{l}\text { B-mode + SE + } \\
\text { FNAB }\end{array}$} & 92,1 & 93,4 & 87,5 & 95,9 & 93 \\
\hline
\end{tabular}

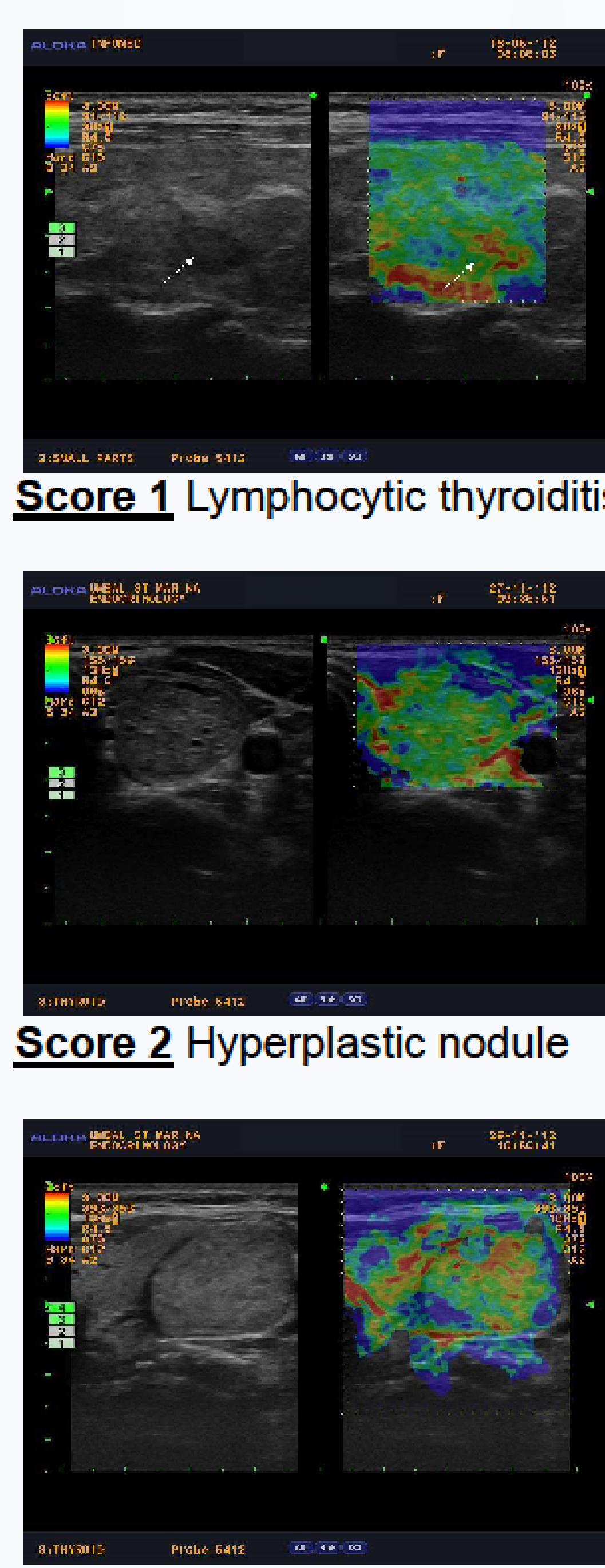

Score 3 Follicular adenoma

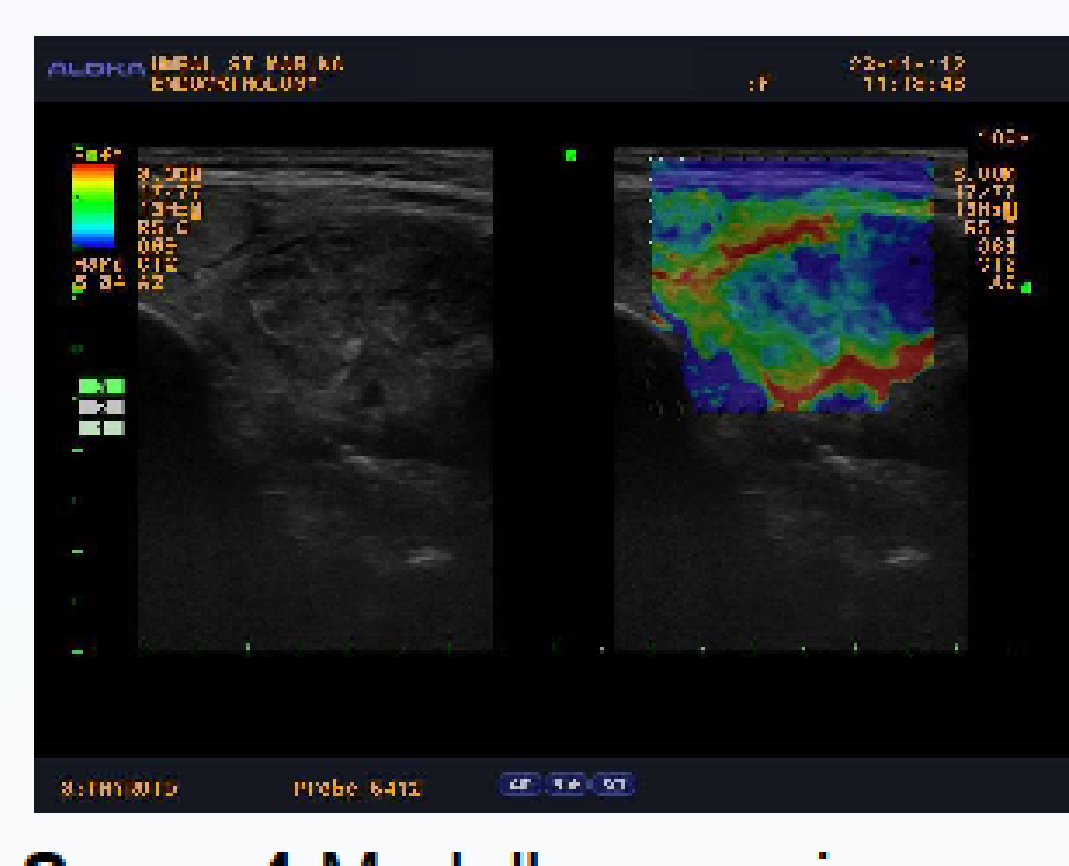

Score 4 Medullary carcinoma

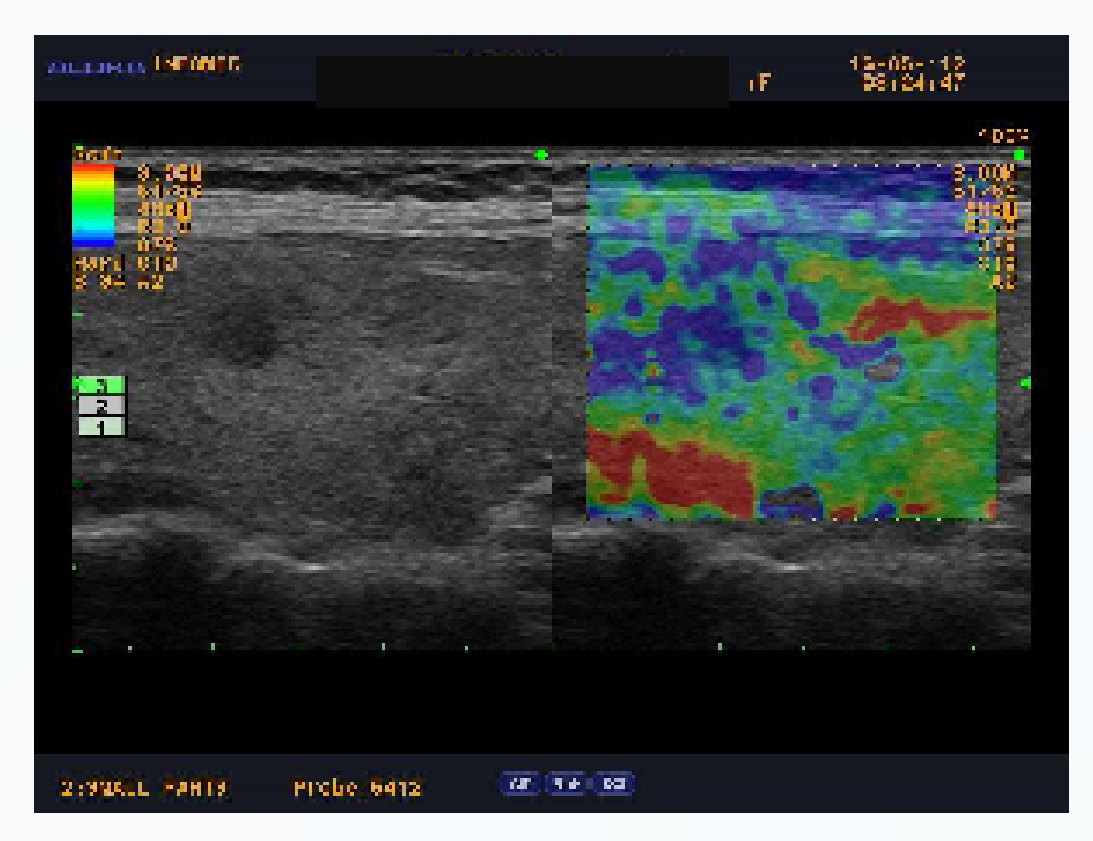

Score 5 Papillary carcinoma

\section{Results}

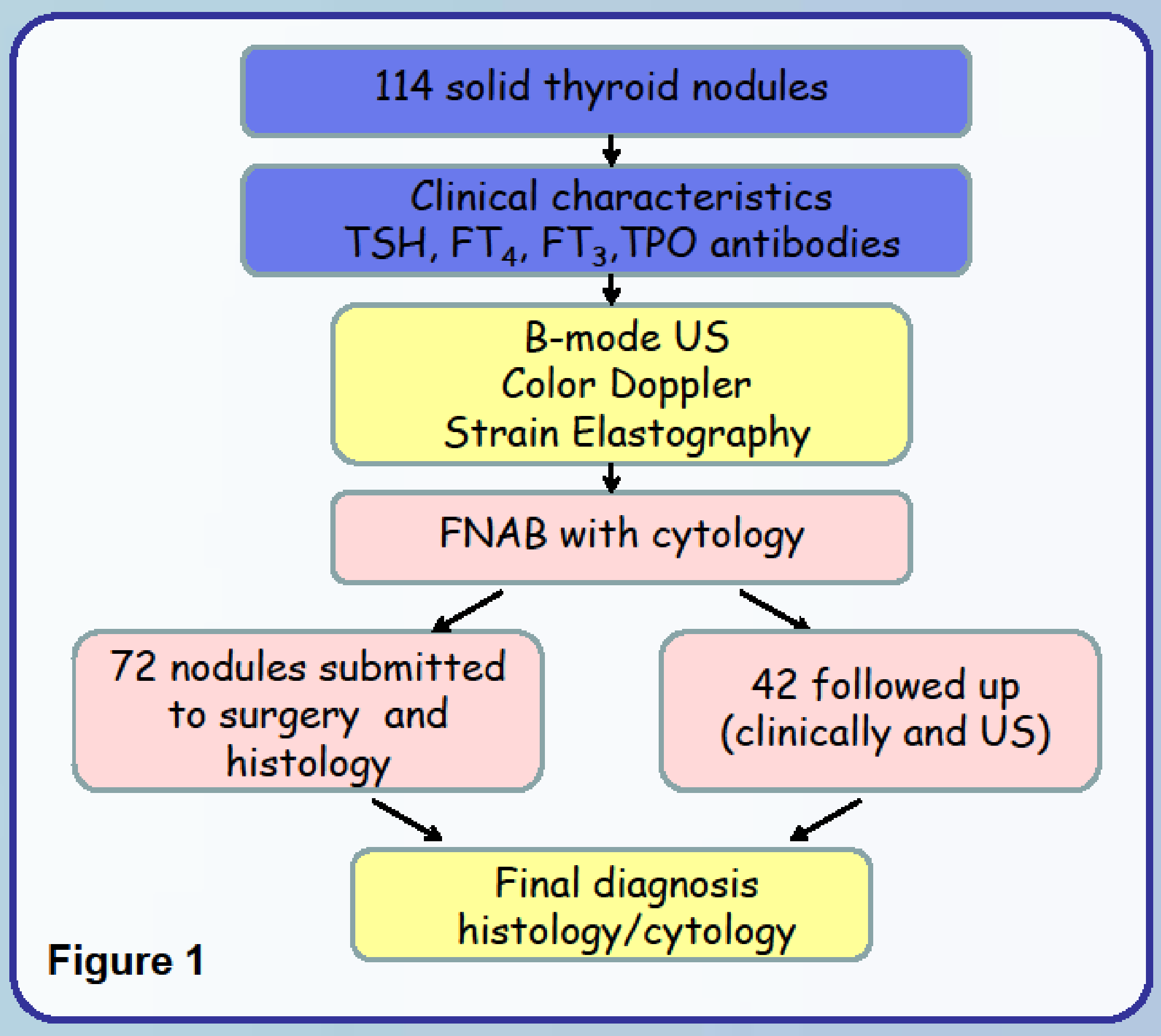

\section{Conclusions}

The high specificity and NPP of SE alone or as an adjunct to conventional US suggests that high elasticity (score 1 and 2) is a promising criterion for excluding malignancy and that elastography may limit the indications for FNAB. Combination of three methods has the highest diagnostic accuracy in differentiating malignant from benign nodules and permits the clinician exact selection of patients who would benefit from surgery.

\section{References:}

Rago T, Santini F, Scutari M, Pinchera A, Vitti $P$ Elastography: new developments in ultrasound for predicting malignancy in thyroid nodules. J Cli Endocrinol Metab 2007; 92: 2917-2922 Ravazi SA, Hadduck TA, Sadigh $G$ et al. Comparative effectiveness elastographic and B-mode ultrasound criteria for diagnostic discrimination of hyroid nodul benign and malignant thyroid nodules. J Ultrasound Med 2014: 33:495-502 Kwak JY, Kim EK. Ultrasound elastography for thyroid nodules: recen Cantisani V, Grazhdani H, Drakonaki E. Strain US elastography for the characterization of thyroid nodules: advantages and limitation. Int $\mathrm{J}$ Endocrino: 2015:908575. doi: 10.1155/2015/908575 